\title{
A Low-Profile Wideband Dual-Resonance Tri-Port MIMO Antenna
}

\author{
Li Ying Nie, Buon Kiong Lau, Senior Member, IEEE, Hanieh Aliakbari, Shang Xiang, Bao Wang, and \\ Xian Qi Lin, Senior Member, IEEE
}

\begin{abstract}
A low-profile wideband tri-polarized MIMO antenna is proposed in this paper. Based on characteristic mode analysis of a circular patch, four slots are etched symmetrically on the patch to transform the high-order monopole-like modes into broadside modes with lower resonant frequencies. Four sets of shorting pins are also applied to simultaneously adjust the resonant frequencies of the monopole-like modes and broadside fundamental modes of the patch. The effects of the slots and the shorting pins on these modes are analyzed in detail. Three dual-resonance ports with high isolation are achieved by exciting three pairs of orthogonal modes of the patch. The proposed antenna is wideband with respect to its profile (bandwidth/height $=216$ ), as facilitated by the dual-resonance property of each port. Experimental results show that the proposed antenna features high port isolation $(>35 \mathrm{~dB})$ and high gain $(>$ $5.58 \mathrm{~dB})$. The proposed low-profile wideband tri-port antenna can be applied in highly integrated MIMO systems.
\end{abstract}

Index Terms-MIMO systems, circular patch antenna, tri-port antenna, wideband antenna, characteristic mode analysis.

\section{INTRODUCTION}

Multiple-input and multiple-output (MIMO) technology has attracted considerable attention due to its potential to dramatically improve the capacity and reliability of the wireless communication systems in radio channels with multipath propagation, without the need for more spectrum or transmit power [1]. In MIMO systems, the data rates can be improved linearly with the number of antennas, as more antennas can provide more parallel data channels [1]. In order to increase the data rates for new generation mobile systems, the number of the antennas working at the same frequency should be increased. Furthermore, implementing very many antennas in a massive MIMO setup at the base station also enable multiuser communications [2], [3] Therefore, it is crucial to develop compact low-profile multiport antennas, beyond the common two-port antennas, to reduce the total dimensions of MIMO systems. For practicality and good performance, the multiport elements should be low-profile, wideband as well as having high isolation and low correlation.

The aim of this paper is to develop a low-profile wideband tri-port single-patch antenna with high isolation. Polarization or pattern diversities are commonly utilized to achieve high isolation of two-port antennas [4]-[8], while similar concepts were also applied in the development of high-isolation tri-port antennas. For examples, three mutually perpendicular co-located dipole/half-slot antenna elements are used to realize a tri-port antenna in [9]. Isolation of above $18 \mathrm{~dB}$ and low signal correlation between ports are obtained due to the

Manuscript submitted for review on Oct. 22, 2020. This work was supported by the Fundamental Research Funds for the Central Universities (Grant number: JZ2021HGTA0144).

$\mathrm{Li}$ Ying Nie is with the School of Computer Science and Information Engineering, Hefei University of Technology, Hefei 230601, China (*E-mail: liyingnie@sina.com).

Buon Kiong Lau, Hanieh Aliakbari, Shang Xiang are with the Department of Electrical and Information Technology, Lund University, Lund 22100, Sweden.

Bao Wang and Xian Qi Lin are with the EHF Key Lab of Fundamental Science, School of Electronic Engineering, University of Electronic Science and Technology of China, Chengdu 611731, China. orthogonal polarization. Using slit-slot transmission lines, the isolation in [10] is improved by $17 \mathrm{~dB}$ compared with conventional tri-polarized slot antenna in [9] . However, for the tri-port antennas in [9] and [10], the three elements used to realize different polarizations are in three perpendicular planes, which leads to the complicated structures and difficulty for antenna integration. Although multiport antennas with lower profile are realized based on multi-layer or coplanar structures in [11]-[15], their bandwidths are narrow and structures need to be further simplified to meet the requirements of the wideband high-integration systems.

All the multiport antennas mentioned above employ the modes produced by more than one constituting antenna structures (e.g., dipole, patch, disk-loaded monopole) to realize multiport characteristics. To realize multiport antennas with less constituting structures, multiple modes can be excited on single-antenna structures [16]-[25]. For example, a single suspended planar metal plate is used to achieve the four-port antennas with polarization and pattern diversity in [16]-[17]. Although large bandwidths are obtained for both antennas, the profiles exceed $0.35 \lambda_{\mathrm{c}}\left(\lambda_{\mathrm{c}}\right.$ is the center frequency of the operating frequency band). To develop low-profile multiport antenna, ring, patch and slot antennas are applied in [18]-[25]. Three different current distributions on the loop are excited to form omnidirectional and dual-polarized broadside radiation patterns in [18]. However, this antenna with profile of $0.24 \lambda_{\mathrm{c}}$ and complicated feeding structure features bandwidth of only $2.8 \%$. Tri-port patch antennas with larger bandwidth are proposed [19]-[22]. However, these antennas focus on providing similar patterns across the three ports, while providing low coupling and correlation. All these antennas feature air gap below the radiating patch and profile higher than $0.1 \lambda_{\mathrm{c}}$. To further reduce the height and structural complexity, two degenerate $\mathrm{TM}_{11}$ modes and one $\mathrm{TM}_{01}$ mode of the circular patch antenna are generated in [23] to realize three orthogonal polarizations with a lower profile $\left(0.083 \lambda_{c}\right)$. Its overlapped bandwidth is more than $13 \%$. Apart from that, single-layer printed slot and patch are utilized in [24]-[25] to develop tri-polarized MIMO antennas with lower profiles. Although high isolations (> 20 $\mathrm{dB}$ ) are obtained due to the modal orthogonality in [18], [23]-[24], each of the ports can excite only one mode (i.e., single resonance), which limits the bandwidth of the tri-port antennas.

To realize a low-profile wideband tri-port antenna using a single element, such as a slot or patch antenna, more modes should be excited by each port. In this paper, a low-profile dual-resonance tri-polarized MIMO antenna based on single circular patch element is proposed. To generate more useful modes without introducing additional structures, four slots are symmetrically etched on the edge of the patch to transform the irrelevant monopole-like higher order modes into desired broadside modes with lower resonant frequencies. Four sets of shorting pins are applied to simultaneously adjust the resonant frequencies of the monopole-like modes and broadside fundamental modes of the patch over the frequency band of interest. Dual-resonance broadside patterns with horizontal polarization are realized by exciting degenerate $\mathbf{J}_{1} / \mathbf{J}_{2}$ and $\mathbf{J}_{6} / \mathbf{J}_{7}$ modes with T-junctions. The monopole-like pattern with vertical polarization is obtained by applying the $\mathbf{J}_{0}$ and $\mathbf{J}_{5}$ modes. High isolation of any two ports is achieved due to the inherent polarization and pattern diversities 
offered by the orthogonal modes. The operating principle and parametric study are elaborated, while measurements of the fabricated prototype were carried out to validate the proposed design. The experimental results show that the proposed antenna has a larger bandwidth-to-height ratio than other low-profile $\left(<0.1 \lambda_{\mathrm{c}}\right)$ tri-port antennas. Meanwhile, high isolation $(>35 \mathrm{~dB})$ and gain $(>5.58 \mathrm{~dB})$ are also obtained within the operating frequency band.

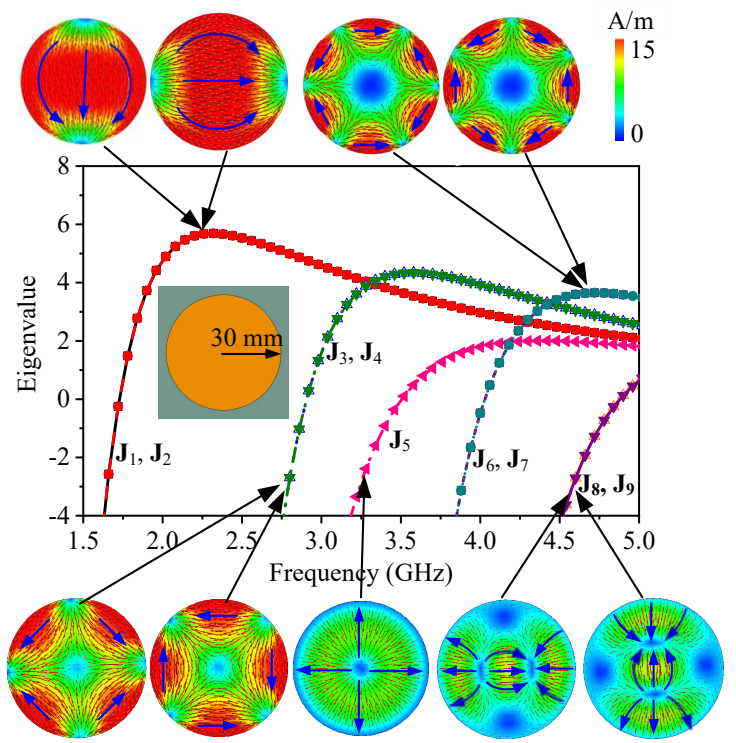

Fig. 1. Characteristic eigenvalues of a circular patch and the corresponding characteristic current distributions at their resonant frequencies.

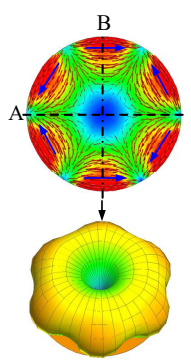

(a)

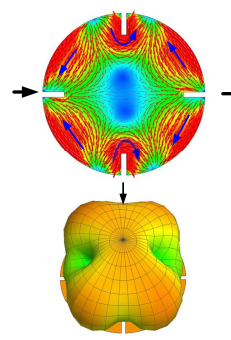

(b)

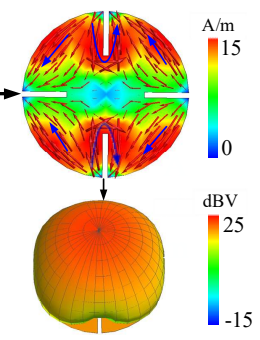

(c)
Fig. 2. Current distributions of (a) the $\mathbf{J}_{6}$ mode without slots, (b) the $\mathbf{J}_{6}$ mode with $8 \mathrm{~mm}$ long slots, (c) the $\mathbf{J}_{6}$ mode with $16 \mathrm{~mm}$ long slots.

\section{Multimode Antenna Design}

\section{A. Modal Analysis and Selection of the Circular Patch}

The theory of characteristic mode (TCM) can provide physical insights into the operating mechanisms of antenna. Therefore, it has been widely applied in the design of multimode antennas [17]. The characteristic modes (CMs) of a circular patch with the radius of 30 $\mathrm{mm}$ printed on one side of a double-sided PCB (with F4B substrate of relative permittivity $\mathcal{E}_{r}=2.55$, loss tangent $\tan \delta=0.002$, and height $h$ $=4 \mathrm{~mm}$ ) were analyzed and the desired modes to realize the tri-port antenna were chosen. All the $\mathrm{CM}$ simulations in this section were done with Altair FEKO (2017 Version). It is noted that lossless substrate was assumed in these simulations, since the CM feature of this software does not account for ohmic losses and low-loss substrate. Infinite ground and infinite substrate layer were also assumed in the $\mathrm{CM}$ simulations to avoid dealing with many finite-ground and finite-substrate based $\mathrm{CMs}$, which will complicate CMA. These assumptions were validated to have limited impact on the results.
Figure 1 shows the characteristic eigenvalues of the circular patch over 1.5-5 GHz and some of the corresponding characteristic currents at their resonant frequencies. It is noted that the characteristic mode analysis (CMA) is performed for the circular patch, without any shorting pin, even though it is known that shorting pin(s) can produce the new mode of $\mathrm{TM}_{01}\left(\mathbf{J}_{0}\right)$. This is because, depending on their locations, the shorting pin can greatly affect the available modes of the circular patch structure. Therefore, more modes are available to be chosen for antenna design by avoiding the pin(s) in the initial analysis. According to the simulated results, except for the $\mathbf{J}_{1} / \mathbf{J}_{2}$ and $\mathbf{J}_{8} / \mathbf{J}_{9}$ modes, all the characteristic modes $\left(\mathbf{J}_{3} / 4, \mathbf{J}_{5}\right.$ and $\left.\mathbf{J}_{6} / \mathbf{J}_{7}\right)$ have monopole-like far fields. The patterns of $\mathbf{J}_{3} / \mathbf{J}_{4}$ and $\mathbf{J}_{6} / \mathbf{J}_{7}$ are not as round (or circular) as that of $\mathbf{J}_{5}$ in the horizontal plane, due to the nonuniform current distributions of these modes along the phi direction (see Fig. 1). Since the resonances of the $\mathrm{TM}_{11}\left(\mathbf{J}_{1} / \mathbf{J}_{2}\right)$ and $\mathrm{TM}_{12}\left(\mathbf{J}_{8} / \mathbf{J}_{9}\right)$ modes of a circular patch are far away from each other and not easy to be combined, they were used to realize a dual-band broadside antenna in [26]. Inspired by the sidelobe-reduction technique for the $\mathrm{TM}_{03}$ mode of the square patch in [27], slots are applied in this paper to modify the $\mathbf{J}_{6} / \mathbf{J}_{7}$ mode of the circular patch to a broadside mode.

The current distributions and corresponding radiation patterns of the modes on the patch with and without four slots are shown in Fig. 2. For the initial circular patch without slots, the radiation pattern of $\mathbf{J}_{6}$ is monopole-like, as shown in Fig. 2(a). After four slots with the width of $1.1 \mathrm{~mm}$ are introduced, the currents crossing the symmetric line B (dark dashed-dotted line in Fig. 2(a)) of the circular patch are cut by the slots, while their contributions to the far field are largely mitigated. However, the symmetric line A (dashed line in Fig. 2(a)) is located along the null of the currents. Therefore, the slots etched on the patch along line A will not affect the currents distribution. The remaining currents on the patch will generate a horizontally polarized broadside pattern with sidelobes as shown in Fig. 2(b). The nulls along symmetric line A disappear when the length is increased to $16 \mathrm{~mm}$ (see Fig. 2(c)), because more currents crossing symmetric line B will be cut and have little contribution to far-field radiation. For $\mathbf{J}_{7}$, the effects of the slots on the symmetric lines A and B will be reversed due to it being orthogonality to the $\mathbf{J}_{6}$ mode.

To realize a tri-port MIMO antenna, shorting pins were used to adjust the $\mathrm{TM}_{02}\left(\mathbf{J}_{5}\right)$ mode and the two degenerate $\mathrm{TM}_{11}\left(\mathbf{J}_{1} / \mathbf{J}_{2}\right)$ modes to resonant at the same frequency in [25]. However, each of the three ports can only excite a single mode, which limits the bandwidth of the antenna. According to the analyses above, since the patterns of the degenerate modes of $\mathbf{J}_{1} / \mathbf{J}_{2}$ and the modified $\mathbf{J}_{6} / \mathbf{J}_{7}$ are all broadside, they can be combined to design two orthogonal broadside dual-resonance ports. In addition, a monopolar circular patch has the potential for realizing a vertical polarized monopole-like radiation pattern based on the $\mathrm{TM}_{01}\left(\mathbf{J}_{0}\right)$ and $\mathrm{TM}_{02}\left(\mathbf{J}_{5}\right)$ modes, despite being low profile [15], [28]-[31]. The $\mathbf{J}_{0}$ mode does not appear in Fig. 1 since it is only available when shorting pin(s) are introduced in a circular patch [30]. In this paper, shorting pins will be introduced to introduce the $\mathbf{J}_{0}$ mode and make the resonant frequency of the $\mathbf{J}_{1} / \mathbf{J}_{2}$ mode closer to that of the $\mathbf{J}_{6} / \mathbf{J}_{7}$ mode. Therefore, dual-resonance radiation for all ports of the tri-port antenna can be realized using the $\mathbf{J}_{0}, \mathbf{J}_{5}, \mathbf{J}_{1} / \mathbf{J}_{2}$, and $\mathbf{J}_{6} / \mathbf{J}_{7}$ modes. However, shorting pins are not included in the initial CMA of the patch to avoid their effects on the other original modes.

\section{B. Mode Adjustments of the Circular Patch}

According to the analyses in part A, four slots etched symmetrically on the edge of the patch were introduced to change the pattern of the $\mathbf{J}_{6} / \mathbf{J}_{7}$ mode from monopole-like to broadside. Apart from their effect on the shape of the pattern, the slots also shifted the eigenvalue curve 
of the $\mathbf{J}_{6} / \mathbf{J}_{7}$ mode in frequency. Table I shows the resonant frequencies of $\mathbf{J}_{6} / \mathbf{J}_{7}$ when the length of the slot is changed from 6 to $16 \mathrm{~mm}$. With the increase of the slot length, the resonant frequency of the $\mathbf{J}_{6} / \mathbf{J}_{7}$ mode shifts downward from 3.79 to $3.1 \mathrm{GHz}$.

According to the current distributions in Fig. 1, the introduced slots also change the current path of the $\mathbf{J}_{1} / \mathbf{J}_{2}$ mode. The simulation results show that the resonant frequency of the $\mathbf{J}_{1} / \mathbf{J}_{2}$ mode shifts from 1.67 to $1.46 \mathrm{GHz}$ as the length of the slot is increased from 6 to $16 \mathrm{~mm}$. The $\mathbf{J}_{5}$ mode is unaffected since its modal currents are in the same directions with the slots.

TABLE I

\begin{tabular}{ccccccc}
\multicolumn{1}{c}{ RESONANT FREQUENCIES OF $\mathbf{J}_{6} / \mathbf{J}_{7}$ WITH DIFFERENT SLOT LENGTHS } \\
\hline \hline Length of the slot (mm) & 6 & 8 & 10 & 12 & 14 & 16 \\
\hline $\begin{array}{c}\text { Resonant frequency } \\
\text { of } \mathbf{J}_{6} / \mathbf{J}_{7}(\mathrm{GHz})\end{array}$ & 3.79 & 3.66 & 3.45 & 3.35 & 3.21 & 3.1 \\
\hline \hline
\end{tabular}

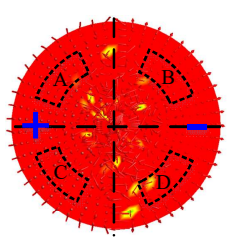

(a)

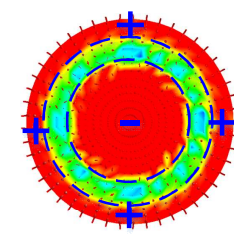

(b)

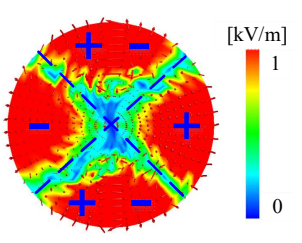

(c)
Fig. 3. Electric field distributions ( $2 \mathrm{~mm}$ below the patch) of (a) $\mathbf{J}_{1}$, (b) $\mathbf{J}_{5}$, and (c) $\mathbf{J}_{6}$. Length and width of each slot along the black dashed lines in subfigure (a) are 16 and $1.1 \mathrm{~mm}$, respectively.

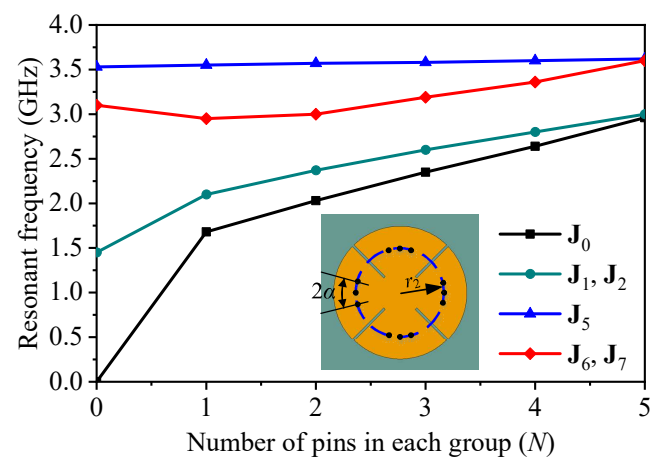

Fig. 4. Effects of the shorting pins on the resonances of $\mathbf{J}_{0}, \mathbf{J}_{1} / \mathbf{J}_{2}, \mathbf{J}_{5}$, and $\mathbf{J}_{6} / \mathbf{J}_{7}$ modes when the length of the slot is $20 \mathrm{~mm}$ and $r_{2}=20 \mathrm{~mm}, \alpha=22^{\circ}$.

To draw the resonant frequencies of the $\mathbf{J}_{1} / \mathbf{J}_{2}$ and $\mathbf{J}_{6} / \mathbf{J}_{7}$ modes closer to each other, shorting pins were introduced to increase the resonant frequency of the $\mathbf{J}_{1} / \mathbf{J}_{2}$ mode. Meanwhile, $\mathrm{TM}_{01}\left(\mathbf{J}_{0}\right)$ mode with non-zero resonant frequency will be generated by these shorting pins [30]. Since the electric field distributions of the $\mathbf{J}_{2}$ and $\mathbf{J}_{7}$ modes can be obtained by rotating the electric field distributions of the $\mathbf{J}_{1}$ and $\mathbf{J}_{6}$ modes, only the electric distributions of the $\mathbf{J}_{1}, \mathbf{J}_{6}$ and $\mathbf{J}_{5}$ modes are shown in Fig. 3. According to the electric field distributions in Fig. 3, to minimize the effects on the $\mathbf{J}_{6} / \mathbf{J}_{7}$ and $\mathbf{J}_{5}$ modes, these shorting pins should be loaded in regions A to D where the electric fields are weak for both $\mathbf{J}_{6} / \mathbf{J}_{7}$ and $\mathbf{J}_{5}$ but strong for $\mathbf{J}_{1} / \mathbf{J}_{2}$. Since the electric fields under the patch for $\mathbf{J}_{0}$ are uniform, its resonant frequency will also increase.

The effects of the shorting pins on the resonances of these modes are also investigated. As shown in Fig. 4, the resonant frequencies of the $\mathbf{J}_{1} / \mathbf{J}_{2}$ and $\mathbf{J}_{0}$ modes increase with the increase in the number of the shorting pins since the shorting pins are located in the regions where the electric fields are strong. Based on the analyses above, the resonances of the $\mathbf{J}_{6} / \mathbf{J}_{7}$ and $\mathbf{J}_{5}$ modes should not be affected by the shorting pins. For $\mathbf{J}_{6} / \mathbf{J}_{7}$ mode, its field is partly affected by the shorting pins when $N>2$, causing a slight increase of its resonant frequency.

\section{The Proposed Tri-Port Antenna and Feeding Network Design}

Based on the analyses above, a tri-port antenna is proposed in this part. The 3D view of the proposed tri-port antenna is shown in Fig. 5. It consists of a circular patch etched with four symmetric slots, a ground plane, four groups of shorting pins, a feeding coaxial cable, and two identical feeding networks for two orthogonal broadside ports (Ports 1 and 2). The circular patch and the ground plane are printed on the top and bottom sides of the $2^{\text {nd }}$ substrate layer (F4B with $\varepsilon_{r}=2.55, \tan \delta=$ 0.002 and $h_{2}=4 \mathrm{~mm}$ ), respectively. Four groups of shorting pins with five pins in each group are symmetrically located on the same circle. The feeding network for each broadside port consists of two identical coupling stubs printed on the top side of the $1^{\text {st }}$ substrate layer and a $\mathrm{T}$-junction printed on the bottom of the $3^{\text {rd }}$ substrate layer. Rogers RO4350 with $\varepsilon_{r}=3.66, \tan \delta=0.004$, and $h_{1}=0.254 \mathrm{~mm}$ is used for both the $1^{\text {st }}$ and $3^{\text {rd }}$ substrate layers. Four metal probes with the radius of $0.25 \mathrm{~mm}$, passing through four shorting pins, are utilized to connect

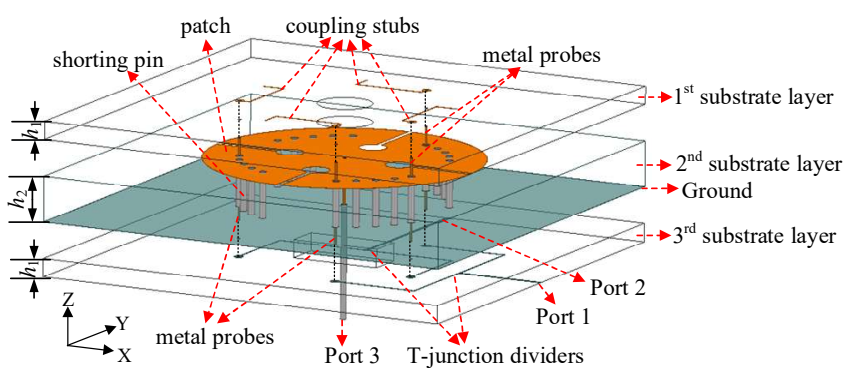

Fig. 5. 3D view of the proposed tri-port antenna.
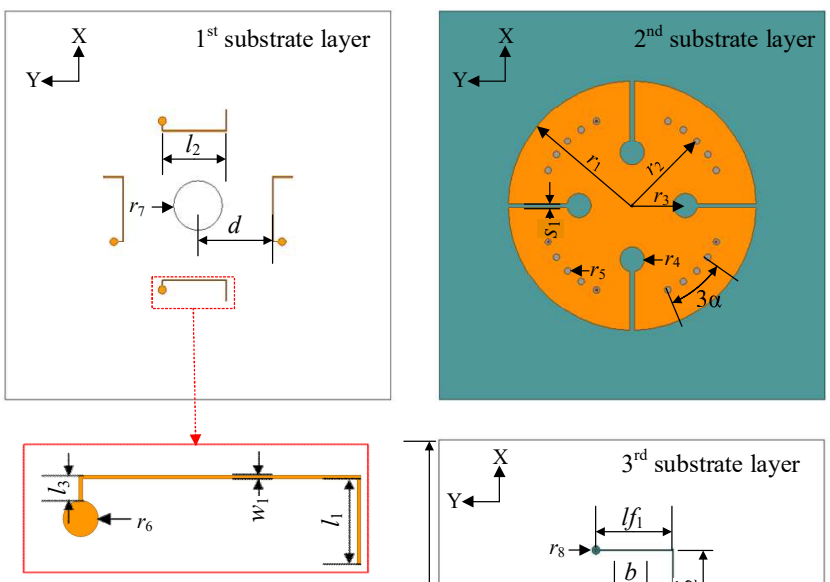

top bottom

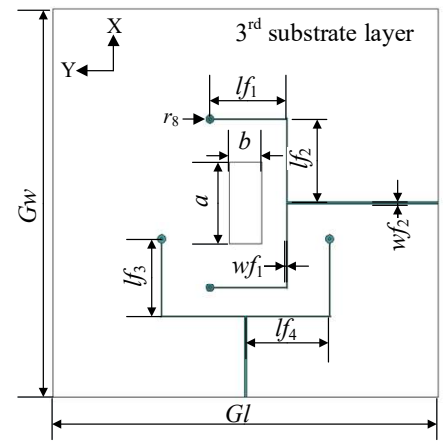

Fig. 6. Top views of the three boards. $\left(G w=G l=110 \mathrm{~mm}, l_{1}=4.8 \mathrm{~mm}, l_{2}=\right.$ $15.9 \mathrm{~mm}, l_{3}=1.4 \mathrm{~mm}, w_{1}=0.2 \mathrm{~mm}, d=18.3 \mathrm{~mm}, r_{1}=30.5 \mathrm{~mm}, r_{2}=22.5 \mathrm{~mm}$, $r_{3}=13.1 \mathrm{~mm}, r_{4}=3 \mathrm{~mm}, r_{5}=0.8 \mathrm{~mm}, r_{6}=1 \mathrm{~mm}, r_{7}=10 \mathrm{~mm}, r_{8}=1 \mathrm{~mm}, s_{1}=$ $1.1 \mathrm{~mm}, \alpha=22^{\circ}, l f_{1}=l f_{3}=19.1 \mathrm{~mm}, l f_{2}=l f_{4}=20.5 \mathrm{~mm}, w f_{1}=0.2 \mathrm{~mm}, w f_{2}=0.6$ $\mathrm{mm}, \mathrm{a}=\mathrm{mm}, \mathrm{b}=\mathrm{mm}$ )

the T-junctions to the coupling stubs. The feeding coaxial cable (for Port 3) located in the middle of the patch is used to excite the $\mathbf{J}_{0}$ and $\mathbf{J}_{5}$ modes with its inner and outer conductors connected to the patch and 
ground plane, respectively. The top views of the three boards and the detailed dimensions are shown in Fig. 6.

To improve the impedance matching of Ports 1 and 2, the four edged slots are terminated with circular slots with radius of $r_{4}$. The resonant frequency of the $\mathbf{J}_{6} / \mathbf{J}_{7}$ mode will decrease with an increase in $r_{4}$, which results in the lower reflection coefficient in the center frequency of Port 1 (shown in Fig. 7). Meanwhile, the impedance matching of Port 3 will start to deteriorate within the overlapped band as $r_{4}$ increased. The optimal value of $r_{4}$ is $3 \mathrm{~mm}$. Beside the circular slots, the microstrip transmission lines with impedance of $80 \Omega$ connecting the T-junction and coupling stubs also contribute to the impedance matching improvements of Ports 1 and 2.

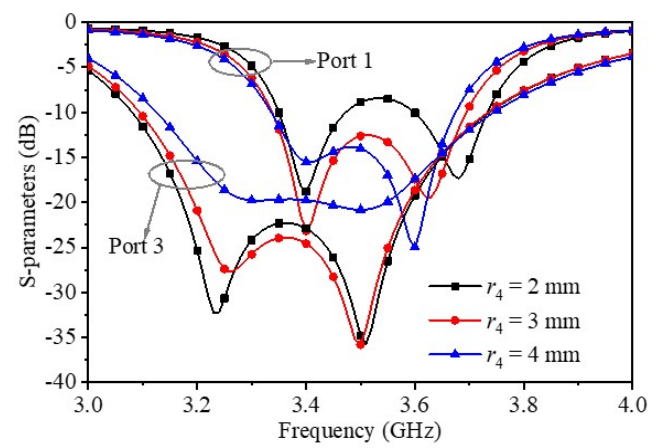

Fig. 7. Effects of the radius of the slots.

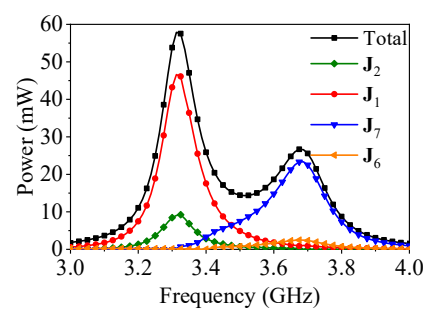

(a)

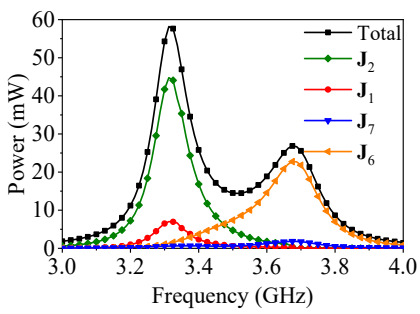

(b)

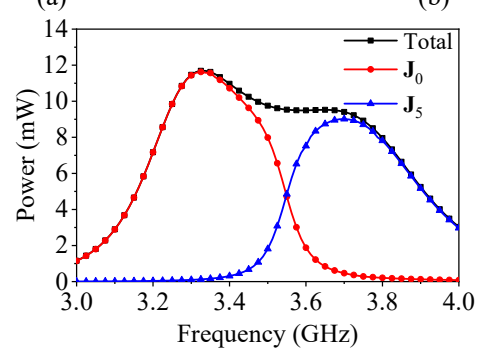

(c)

Fig. 8. Modal far-field radiated powers of the proposed dual-resonance tri-port antenna of (a) Port 1, (b) Port 2, and (c) Port 3.

To realize the feeding networks of Ports 1 and 2, the Rogers RO4350 substrate (with thickness $h_{1}$ ) is arranged on the top of the patch. As a result, the resonances of the corresponding modes will be slightly changed compared with those obtained without the top substrate in the modal analysis in Section II-A. The dual-resonance property of the proposed tri-port antenna without $\mathrm{T}$-junctions and metal probes is clearly revealed by calculating the modal radiated powers using Altair FEKO (with the top substrate added to the CM analysis model, also assumed to be lossless and infinite). Figure 8 shows the total far-field radiated power and the radiated power of each mode. Four voltage sources (set to 1-volt) are added between the coupling stubs and the patch. The modes with radiated power below $0.3 \mathrm{~mW}$ are not shown. As shown in Fig. 8(a), the antenna radiation of Port 1 is dominated by $\mathbf{J}_{1}$ and $\mathbf{J}_{7}$. The main contribution to the total radiation comes from the
$\mathbf{J}_{2}$ and $\mathbf{J}_{6}$ modes for Port 2 (Fig. 8(b)). For Port 3, excited by the fifth voltage source (also set to 1-volt) set between the center of the patch and the ground plane, the $\mathbf{J}_{0}$ and $\mathbf{J}_{7}$ modes contribute to the far-field radiation at the lower and higher frequencies, respectively (see Fig. $8(\mathrm{c})$ ). Therefore, the desired modes for the three ports have been selectively excited by the proposed excitation scheme.

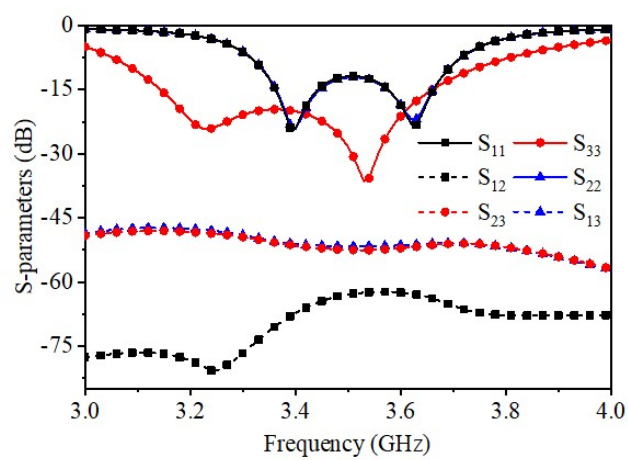

Fig. 9. Simulated S-parameters of the proposed tri-port antenna.

The simulated S-parameters of the proposed antenna (with the finite substrate/ground plane, complete feeding network and ohmic losses) are obtained using full-wave simulation in 2019 Ansys HFSS and shown in Fig. 9. The overlapped operating frequency range of these three ports is from 3.34 to $3.69 \mathrm{GHz}$, for the $10 \mathrm{~dB}$ return loss. The isolation of any two ports is higher than $50 \mathrm{~dB}$ within the working band.

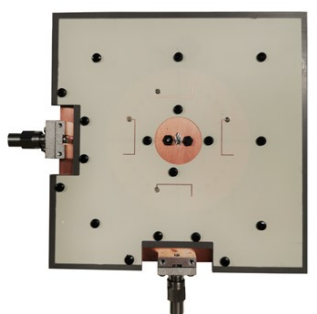

(a)

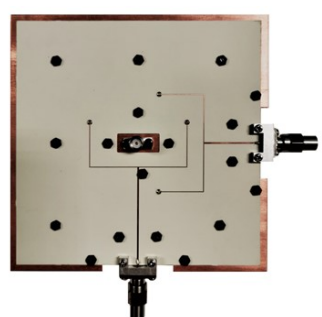

(b)
Fig. 10. Photographs of the fabricated tri-port antenna: (a) front view, (b) back view.

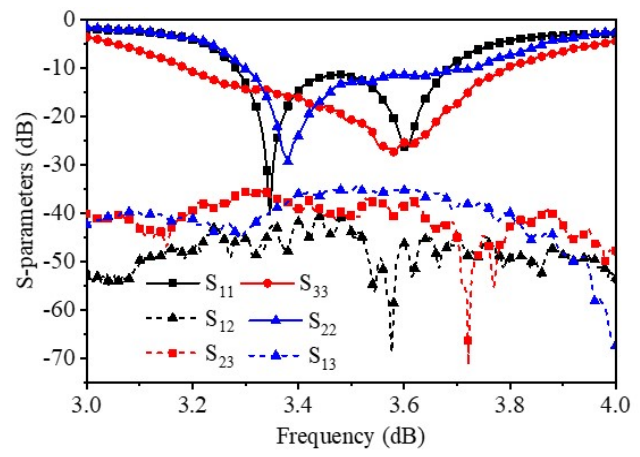

Fig. 11. Measured S-parameters of the proposed tri-port antenna

\section{MEASUREMENT AND DISCUSSION}

The proposed antenna was fabricated and measured. Photographs of the fabricated tri-port antenna are shown in Fig. 10. Measured results of the S-parameters in Fig. 11 show that the overlapped working frequency range of the three ports is from 3.3 to $3.68 \mathrm{GHz}$. The isolation of any two ports is higher than $35 \mathrm{~dB}$ within the frequency 


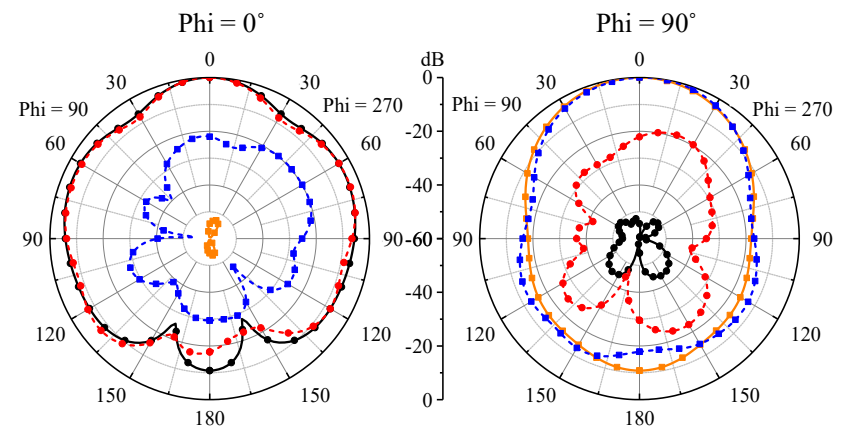

(a)

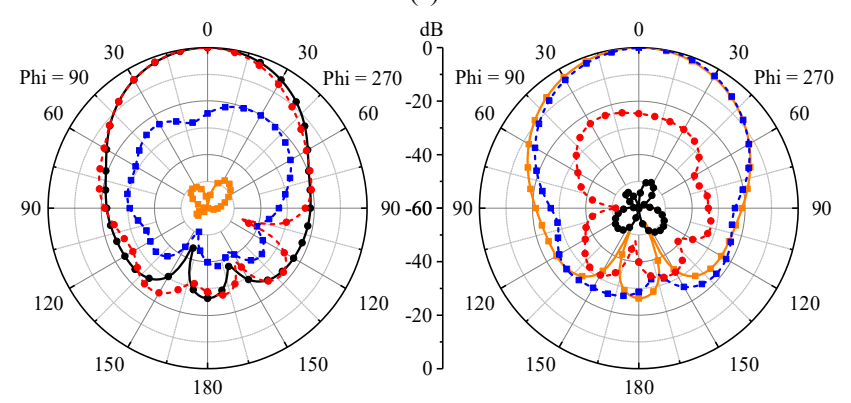

(b)

$$
\longrightarrow \text { Sim. } G_{\text {phi }} \longrightarrow \text { Sim. } G_{\text {theta }} \text {---- Meas. } G_{\text {phi }} \text {-- }- \text { Meas. } G_{\text {theta }}
$$

Fig. 12. Simulated and measured normalized radiation patterns of the proposed tri-port antenna when Port 1 is excited with other two ports $50 \Omega$ matched at (a) $3.33 \mathrm{GHz}$ and (b) $3.68 \mathrm{GHz}$.
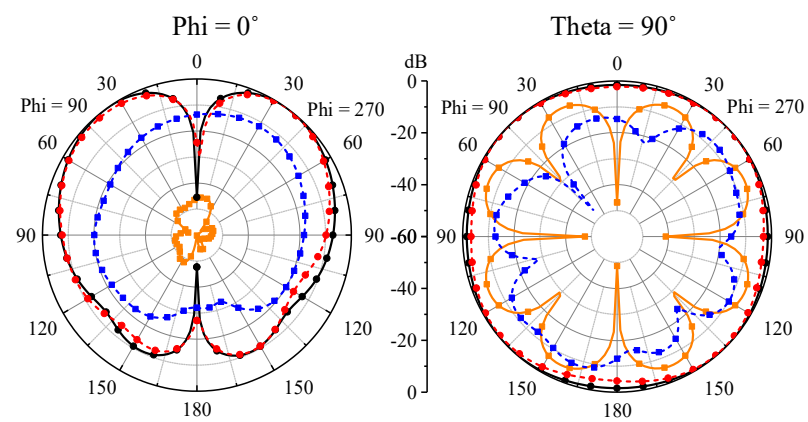

(a)
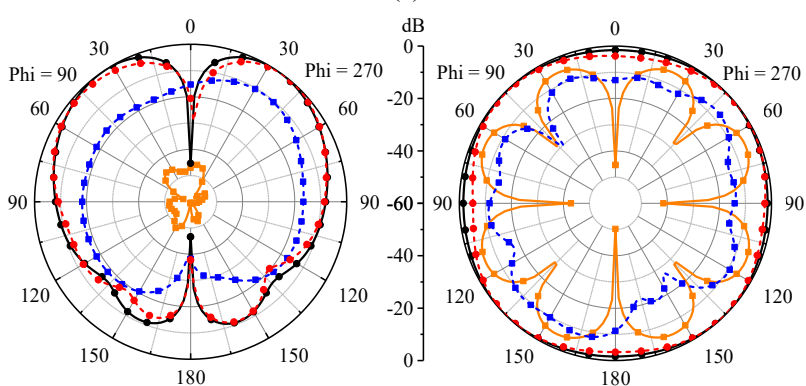

(b)

$\longrightarrow$ Sim. $G_{\text {phi }} \longrightarrow$ Sim. $G_{\text {theta }} \cdots--$ Meas. $G_{\text {phi }}$ - - - - Meas. $G_{\text {theta }}$

Fig. 13. Simulated and measured normalized radiation patterns of the proposed tri-port antenna when Port 3 is excited with other two ports $50 \Omega$ matched at (a) $3.33 \mathrm{GHz}$ and $3.68 \mathrm{GHz}$.

band. The discrepancies between the simulated and measured results (such as asymmetry in the measured $S_{11}$ and $S_{22}$ ) are caused by fabrication and assembling errors, such as the radius of the shorting pins and tiny gaps between different substrate layers.
Figures 12 and 13 show the normalized radiation patterns of the proposed antenna when Ports 1 and 3 are excited, respectively. The reference coordinate system is the same as that in Fig. 5. The radiation pattern is broadside when Port 1 is excited. The two modes excited by Port 1 are the degenerate modes of those excited by Port 2 . Therefore, the radiation patterns of Ports 1 and 2 are the same, except for the polarization. Conical beam is obtained when Port 3 is excited. Measured main polarizations agree well with the simulated ones. Due to tolerances in the measurement setup, the measured cross polarization is higher than the simulated one. In general, the measured and simulated realized peak gains given in Fig. 14 agrees well with each other. However, the discrepancy can be up to $10 \%$ at some frequencies. This is due to bigger dielectric loss, fabrication tolerance of the multilayer PCB, imperfect soldering and measurement tolerances. The measured realized peak gains of the three ports are higher than $5.58 \mathrm{~dB}$ within the overlapped bandwidth (3.3 to 3.68 $\mathrm{GHz})$.

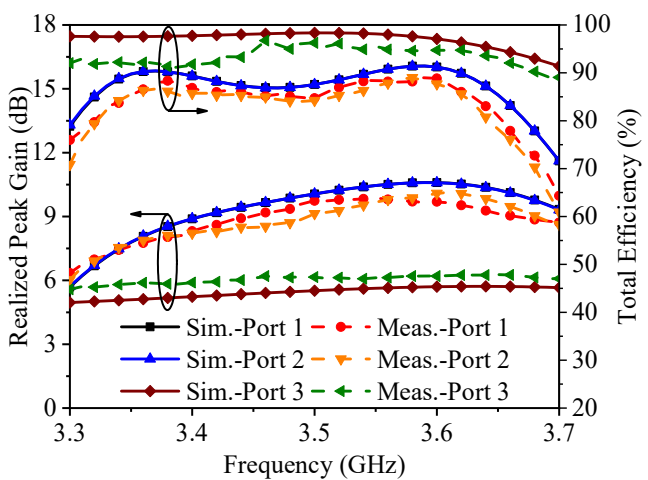

Fig. 14. Simulated and measured realized peak gains and total efficiencies of the proposed tri-port antenna.

TABLE II

COMPARISON OF LOW-PROFILE TRI-PORT MIMO ANTENNAS

\begin{tabular}{|c|c|c|c|c|c|c|c|}
\hline Ref. & $\begin{array}{c}\text { Basic } \\
\text { structure }\end{array}$ & $\begin{array}{c}\text { Structural } \\
\text { complexity }\end{array}$ & \begin{tabular}{|l} 
Iso. \\
(dB)
\end{tabular} & \begin{tabular}{|l|} 
BW \\
$(\%)$
\end{tabular} & $\begin{array}{c}\text { Size* } \\
\left(\lambda_{\mathrm{c}}\right)\end{array}$ & $\begin{array}{c}\mathrm{H} \\
\left(\lambda_{\mathrm{c}}\right)\end{array}$ & $\begin{array}{c}\mathrm{BW} \\
/ \mathrm{H}\end{array}$ \\
\hline$[12]$ & $\begin{array}{l}\text { Ring patch; } \\
\text { top-loaded } \\
\text { monopole }\end{array}$ & Medium & $>24$ & 4.5 & $D=0.35$ & 0.047 & 95.7 \\
\hline$[15]$ & $\begin{array}{l}\text { Patch; dipole } \\
\text { \& patch }\end{array}$ & High & $>31$ & 14 & $D=0.92$ & 0.09 & 155.6 \\
\hline$[23]$ & Single patch & Medium & $>20$ & 1.4 & $D=0.10$ & 0.076 & 18.4 \\
\hline [24] & $\begin{array}{l}\text { Patch with } \\
\text { slots }\end{array}$ & Medium & $>35$ & 2.3 & $L=0.81$ & 0.062 & 37.1 \\
\hline$[25]$ & Single patch & Low & $>13$ & 3.4 & $D=0.58$ & 0.035 & 97.1 \\
\hline $\begin{array}{l}\text { This } \\
\text { work }\end{array}$ & Single patch & medium & $>35$ & 10.8 & $D=0.71$ & 0.05 & 216 \\
\hline
\end{tabular}

$\lambda_{\mathrm{c}}$ : Wavelength evaluated at the center frequency of the overlapped bandwidth; Iso.: Isolation; BW: Bandwidth; $\mathrm{H}$ : Total height; *: The size is shown with diameter $(D)$ for circular shape and side length $(L)$ for square shaped. The size of ground plane is not included.

Comparisons between the proposed antenna and previously reported low-profile tri-port antennas are given in Table II. Since the bandwidth of the antenna is limited by its height, the bandwidth-to-height ratio $(\mathrm{BW} / \mathrm{H})$ is calculated to provide a fairer bandwidth comparison. Although the $\mathbf{J}_{0}, \mathbf{J}_{1} / \mathbf{J}_{2}$, and $\mathbf{J}_{5}$ modes are also used separately in other published literatures, a larger overlapped bandwidth-to-height ratio is obtained in this paper by combining these modes with the $\mathbf{J}_{6} / \mathbf{J}_{7}$ mode 
using the slots and shorting pins. In addition, the port isolation of any two ports is high (i.e., measured isolation $>35 \mathrm{~dB}$ ) due to the far-field orthogonality of the utilized modes. Furthermore, high gain and good efficiency (see Fig. 14) are also achieved for the proposed tri-port antenna. Although the proposed tri-layer structure has more structural complexity compared with the single-layer antenna in [24] and [25], it can still be easily fabricated by machining processes of multi-layer board, which cannot be applied to antennas with air layer or vertical feeding networks [12], [15] and [23].

\section{CONCLUSION}

This paper presents a low-profile wideband tri-port MIMO antenna realized by a single patch. The monopole-like pattern of the $\mathbf{J}_{6} / \mathbf{J}_{7}$ mode is changed to be desired broadside by the four slots etched symmetrically on the edge of the patch. Except for the resonance of the $\mathbf{J}_{5}$ mode, the resonant frequencies of the $\mathbf{J}_{0}, \mathbf{J}_{1} / \mathbf{J}_{2}$, and $\mathbf{J}_{6} / \mathbf{J}_{7}$ modes can be tuned by the slots and four sets of the shorting pins. Due to the dual-resonance property, the proposed antenna has a larger bandwidthto-profile ratio compared with other low-profile tri-port antennas in literatures. Moreover, high isolation and low ECC of any the two ports are realized. The proposed tri-port antenna is suitable for application in highly integrated MIMO systems.

\section{REFERENCES}

[1] D. Gesbert, M. Shafi, D. Shiu, P. J. Smith, and A. Naguib, "From theory to practice: An overview of MIMO space-time coded wireless systems," IEEE J. Sel. Area Commun., vol. 21, no. 3, pp. 281-302, Mar. 2003.

[2] F. Rusek, D. Persson, B. K. Lau, E. G. Larsson, O. Edfors, F. Tufvesson, and T. L. Marzetta, "Scaling up MIMO: Opportunities and challenges with very large arrays," IEEE Signal Proc. Mag., vol. 30, no. 1, pp. 40-60, Jan. 2013.

[3] D. Manteuffel and R. Martens, "Compact multimode multielement antenna for indoor UWB massive MIMO," IEEE Trans. Antennas Propag., vol. 64, no. 7, pp. 2689-2697, Jul. 2016.

[4] B. Rohani, K. Takahashi, H. Arai, Y. Kimura, and T. Thara, "Improving channel capacity in indoor $4 \times 4$ MIMO base station utilizing smal bidirectional antenna," IEEE Trans. Antennas Propag., vol. 66, no. 1, pp. 393-400, Jan. 2018

[5] A. Narbudowicz and M. J. Ammann, "Low-cost multimode patch antenna for dual MIMO and enhanced localization use," IEEE Trans. Antennas Propag., vol. 66, no. 1, pp. 405-408, Jan. 2018.

[6] M. V. Komandla, G. Mishra, and S. K. Sharma, "Investigations on dual slant polarized cavity-backed massive MIMO antenna panel with beamforming," IEEE Trans. Antennas Propag., vol. 65, no. 12, pp. 6794-6799, Dec. 2017.

[7] J. Zhu, S. Li, S. Liao, and Q. Xue, "Wideband low-profile highly isolated MIMO antenna with artificial magnetic conductor," IEEE Antennas Wireless Propag. Lett., vol. 17, no. 3, pp. 458-462, March 2018.

[8] S. S. Yang and K. M. Luk, "Design of a wide-band L-probe patch antenna for pattern reconfiguration or diversity applications," IEEE Trans. Antennas Propag., vol. 54, no. 2, pp. 433-438, Feb. 2006.

[9] C. Chiu, J. Yan, and R. D. Murch, "Compact three-port orthogonally polarized MIMO antennas," IEEE Antennas Wireless Propag. Lett., vol. 6, pp. 619-622, Dec. 2007.

[10] L. Liu, C. Liu, Z. Li, X. Yin, and Z. N. Chen, "Slit-slot line and its application to low cross-polarization slot antenna and mutual-coupling suppressed tripolarized MIMO antenna," IEEE Trans. Antennas Propag., vol. 67, no. 1, pp. 4-15, Jan. 2019.

[11] H. Zhong, Z. Zhang, W. Chen, Z. Feng, and M. F. Iskander, "A tripolarization antenna fed by proximity coupling and probe," IEEE Antennas Wireless Propag. Lett., vol. 8, pp. 465-467, 2009.

[12] X. Gao, H. Zhong, Z. Zhang, Z. Feng, and M. F. Iskander, "Low-profile planar tripolarization antenna for WLAN communications," IEEE Antennas Wireless Propag. Lett., vol. 9, pp. 83-86, 2010.

[13] J. Sarrazin, Y. Mahe, S. Avrillon, and S. Toutain, "Collocated microstrip antennas for MIMO systems with a low mutual coupling using mode confinement," IEEE Trans. Antennas Propag., vol. 58, no. 2, pp. 589-592, Feb. 2010.
[14] Y. Wen, D. Yang, H. Zeng, M. Zou, and J. Pan, "Bandwidth enhancement of low-profile microstrip antenna for MIMO applications," IEEE Trans. Antennas Propag., vol. 66, no. 3, pp. 1064-1075, March 2018.

[15] N. Nguyen-Trong, S. X. Ta, M. Ikram, K. Bertling, and A. M. Abbosh, "A low-profile wideband tripolarized antenna," IEEE Trans. Antennas Propag., vol. 67, no. 3, pp. 1946-1951, March 2019.

[16] D. Manteuffel and R. Martens, "Compact multimode multielement antenna for indoor UWB massive MIMO," IEEE Trans. Antennas Propag., vol. 64, no. 7, pp. 2689-2697, July 2016.

[17] W. Su, Q. Zhang, S. Alkaraki, Y. Zhang, X. Zhang, and Y. Gao, "Radiation energy and mutual coupling evaluation for multimode MIMO antenna based on the theory of characteristic mode," IEEE Trans. Antennas Propag., vol. 67, no. 1, pp. 74-84, Jan. 2019.

[18] K. Saurav, N. K. Mallat, and Y. M. M. Antar, "A three-port polarization and pattern diversity ring antenna," IEEE Antennas Wireless Propag. Lett., vol. 17, no. 7, pp. 1324-1328, July 2018.

[19] C.-Y. Chiu, B. K. Lau and R. Murch, "Bandwidth enhancement technique for broadside tri-modal patch antenna," IEEE Open J. Antennas Propag., vol. 1, pp. 524-533, 2020.

[20] C. -Y. Chiu, S. Shen, B. K. Lau and R. Murch, "The design of a trimodal broadside antenna element for compact massive MIMO arrays: utilizing the theory of characteristic modes," IEEE Antennas Propag. Mag., vol. 62, pp. 46-61, Dec. 2020.

[21] K. L. Wong, C. M. Chou, Y. J. Yang, and K. Y. Wang, "Multipolarized wideband circular patch antenna for fifth-generation multi-input-multi-output access-point application," IEEE Antennas Wireless Propag. Lett., vol. 18, pp. 2184-2188, 2019.

[22] K. L. Wong, H. J. Chang, J. Z. Chen, and K. Y. Wang, "Three wideband monopolar patch antennas in a Y-shape structure for 5G multi-input-multi-output access points," IEEE Antennas Wireless Propag. Lett., vol. 19, pp. 393-397, 2020.

[23] Y. Zhang, K. Wei, Z. Zhang, and Z. Feng, "A broadband patch antenna with tripolarization using quasi-cross-slot and capacitive coupling feed," IEEE Antennas Wireless Propag. Lett., vol. 12, pp. 832-835, 2013.

[24] N. P. Lawrence, C. Fumeaux, and D. Abbott, "Planar triorthogonal diversity slot antenna," IEEE Trans. Antennas Propag., vol. 65, no. 3, pp. 1416-1421, March 2017.

[25] D. Piao and Y. Wang, "Tripolarized MIMO antenna using a compact single-layer microstrip patch," IEEE Trans. Antennas Propag., vol. 67, no. 3, pp. 1937-1940, March 2019.

[26] X. Zhang and L. Zhu, "Dual-band high-gain differentially fed circular patch antenna working in $\mathrm{TM}_{11}$ and $\mathrm{TM}_{12}$ modes," IEEE Trans. Antennas Propag., vol. 66, no. 6, pp. 3160-3165, June 2018.

[27] X. Zhang, L. Zhu, and Q. Wu, "Sidelobe-reduced and gain-enhanced square patch antennas with adjustable beamwidth under $\mathrm{TM}_{03}$ mode operation," IEEE Trans. Antennas Propag., vol. 66, no. 4, pp. 1704-1713, April 2018.

[28] Q. Hou, H. Tang, Y. Liu, and X. Zhao, "Dual-frequency and broadband circular patch antennas with a monopole-type pattern based on epsilon-negative transmission line," IEEE Antennas Wireless Propag. Lett., vol. 11, pp. 442-445, 2012.

[29] J. Liu, Q. Xue, H. Wong, H. W. Lai, and Y. Long, "Design and analysis of a low-profile and broadband microstrip monopolar patch antenna," IEEE Trans. Antennas Propag., vol. 61, no. 1, pp. 11-18, Jan. 2013.

[30] W. Lin and H. Wong, "Circularly polarized conical-beam antenna with wide bandwidth and low profile," IEEE Trans. Antennas Propag., vol. 62, no. 12, pp. 5974-5982, Dec. 2014.

[31] S. Liu, W. Wu, and D. Fang, "Wideband monopole-like radiation pattern circular patch antenna with high gain and low cross-polarization," IEEE Trans. Antennas Propag., vol. 64, no. 5, pp. 2042-2045, May 2016. 